\title{
Risk Factors and Outcomes of Non-albicans Candida Bloodstream Infection in Patients with Candidemia at Siriraj Hospital- Thailand's Largest National Tertiary Referral Hospital
}

\author{
Chaiyapong Ngamchokwathana ${ }^{1}$ D, Piriyaporn Chongtrakool ${ }^{2}$, Amiroh Waesamaae ${ }^{3}$ \\ and Methee Chayakulkeeree ${ }^{4, *(D)}$
}

1 Department of Medicine, Faculty of Medicine Siriraj Hospital, Mahidol University, Bangkok 10700, Thailand octofive@gmail.com

2 Department of Microbiology, Faculty of Medicine Siriraj Hospital, Mahidol University, Bangkok 10700, Thailand; piriyaporn.cho@mahidol.ac.th

3 Research Department, Faculty of Medicine Siriraj Hospital, Mahidol University, Bangkok 10700, Thailand; amiroh.wae@gmail.com

4 Department of Medicine, Division of Infectious Diseases and Tropical Medicine, Faculty of Medicine Siriraj Hospital, Mahidol University, Bangkok 10700, Thailand

* Correspondence: methee.cha@mahidol.ac.th; Tel.: +662-4199-462

check for

updates

Citation: Ngamchokwathana, C.; Chongtrakool, P.; Waesamaae, A.; Chayakulkeeree, M. Risk Factors and Outcomes of Non-albicans Candida Bloodstream Infection in Patients with Candidemia at Siriraj Hospital - Thailand's Largest National Tertiary Referral Hospital. J. Fungi 2021, 7, 269 https://doi.org/10.3390/jof7040269

Academic Editor: Ronen Ben-Ami

Received: 23 February 2021

Accepted: 29 March 2021

Published: 1 April 2021

Publisher's Note: MDPI stays neutral with regard to jurisdictional claims in published maps and institutional affiliations.

Copyright: (c) 2021 by the authors. Licensee MDPI, Basel, Switzerland. This article is an open access article distributed under the terms and conditions of the Creative Commons Attribution (CC BY) license (https:/ / creativecommons.org/licenses/by/ $4.0 /)$.

\begin{abstract}
This study aimed to investigate the risk factors for and the outcomes of patients with candidemia caused by non-albicans Candida. Candidemia patients treated at Siriraj Hospital (Bangkok, Thailand) during January 2016 to December 2017 were enrolled. A total of 156 patients (mean age: 65 years, $56.4 \%$ male) were included. The most prevalent underlying conditions were diabetes (32.1\%), chronic cardiac disease $(28.2 \%)$, chronic kidney disease $(26.9 \%)$, and hematologic malignancies (21.2\%). Candida species isolated from patient blood were C. tropicalis (49.4\%), C. albicans (28.8\%), C. glabrata $(16.7 \%)$, and C. parapsilosis (5.1\%). Fluconazole resistance was significantly increased in C. tropicalis $(37.8 \%)$. No independent risk factors were associated with patients with non-albicans Candida candidemia compared to those with C. albicans candidemia. There was no significant difference in mortality between patients with non-albicans Candida candidemia and patients with C. albicans candidemia (OR: 1.35, 95\% CI: 0.64-2.85). When compared with C. albicans candidemia, multivariate analysis revealed chronic liver disease (OR: 11.39, 95\% CI: 1.38-94.02), neutropenia (OR: 4.31, 95\% CI: 1.34-13.87), and male gender (OR: 2.34, 95\% CI: 1.04-5.29) to be independent risk factors for $C$. tropicalis candidemia. The observed high resistance of $C$. tropicalis to fluconazole indicates that fluconazole should not be used for empirical antifungal treatment in these patients.
\end{abstract}

Keywords: risk factors; outcomes; non-albicans Candida; candidiasis; candidemia; Candida; Thailand

\section{Introduction}

Candida spp. is the most common fungal pathogen that causes nosocomial infection [1,2]. Candidemia, which is a blood stream infection caused by Candida, is a serious public health problem that is associated with high mortality and high medical and economic costs [2]. A previous study reported the incidence of candidemia to be approximately 13.3 per 100,000 patients [3], and the mortality rate ranged from $36 \%$ to $59 \%$ [4,5]. The incidence of Candida infection increases following advanced medical interventions, such as hematopoietic stem cell transplantation and solid organ transplantation, as well as after invasive medical procedures, such as the use of vascular catheter or mechanical ventilator [4]. Factors reported to be associated with mortality in candidemia included neutropenia, septic shock, intensive care unit (ICU) admission, inappropriate antifungal therapy within $72 \mathrm{~h}$, and renal failure [5].

Previous epidemiological studies demonstrated the same trend toward increasing numbers of patients with candidemia in both neutropenic and non-neutropenic individuals. 
The incidence of non-albicans Candida has risen dramatically in some countries, especially in Asia, and there has been a shift from Candida albicans to non-albicans Candida species as the more prevalent causative pathogen in candidemia patients [6-11]. Candida tropicalis is the most common non-albicans Candida species in tropical countries, including Thailand [12]. Although fluconazole resistance is commonly found in Candida glabrata [13], C. tropicalis isolates exhibit decreased susceptibility to azole agents [13,14]. The mortality rate in candidemia may vary according to Candida species [15]. However, the mortality rate in candidemia caused by non-albicans Candida is relatively higher than the mortality rate in candidemia caused by $C$. albicans [16]. Early appropriate antifungal treatment significantly improves outcomes, and it reduces the high hospital costs normally associated with candidemia [17]. Delay in the initiation of fluconazole therapy in hospitalized patients with candidemia significantly adversely influenced mortality [18].

Despite the existing data from previous studies, the risk factors and clinical outcomes of patients with bloodstream infection from non-albicans Candida are still unclear. Accordingly, the aim of this study was to identify the independent risk factors for candidemia caused by non-albicans Candida, particularly C. tropicalis. The secondary objective was to investigate antifungal susceptibility and mortality among non-albicans Candida candidemia patients.

\section{Materials and Methods}

\subsection{Study Design}

This retrospective cohort study included candidemia patients diagnosed and treated at the Faculty of Medicine Siriraj Hospital, Mahidol University, Bangkok, Thailand during January 2016 to December 2017. Siriraj Hospital is a 2300-bed national super-tertiary referral center. This study was registered in the Thai Clinical Trials Registry (ID: TCTR20190501003).

\subsection{Study Population}

All candidemia patients aged 18 years or older who had a positive blood culture for Candida spp. were enrolled. Patients whose blood culture grew more than 1 species of Candida, who had recurrent candidemia, and/or who had an incomplete medical record were excluded.

Collected patient demographic and clinical data included age; gender; duration of hospital stay; and risk factors for candidemia, including central venous catheter, mechanical ventilation, parenteral nutrition, hemodialysis, peritoneal dialysis, recent abdominal surgery, ICU admission, urinary catheter, prosthesis, broad spectrum antibiotics, corticosteroid equivalent to prednisolone at least $20 \mathrm{mg}$ /day for at least 2 weeks, absolute neutrophil count $<500$ cells $/ \mathrm{mm}^{3}$, yeast in non-sterile body samples, prior antifungal exposure, and comorbidities. Candidemia was categorized into primary fungemia, acute disseminated candidiasis, catheter-related blood stream infection, intra-abdominal infection, hepatosplenic abscess, bone and joint infection, central nervous system infection, endocarditis, and urinary tract infection. All Candida isolated from blood cultures were identified to the species level. The species identification of Candida was performed based on biochemical characteristics using chromogenic medium (Brilliance ${ }^{\mathrm{TM}}$ Candida agar) and RapID $^{\mathrm{TM}}$ Yeast Plus Panel (Thermo Fisher Scientific, Kent, UK). Antifungal susceptibility was tested by the microbroth dilution method using Thermo Scientific ${ }^{\mathrm{TM}}$ Sensititre ${ }^{\mathrm{TM}}$ YeastOne ${ }^{\mathrm{TM}}$ YO9 AST Plate (Thermo Fisher Scientific, Waltham, MA, USA). Minimal inhibitory concentration (MIC) breakpoints were interpreted according to the Clinical Laboratory Standards Institute (CLSI). Antifungal susceptibility test results and clinical outcomes were analyzed.

\subsection{Statistical Analysis}

Continuous data are presented as mean \pm standard deviation (SD) for normally distributed data, and as median and interquartile range (IQR) for non-normally distributed data. Categorical data are presented as frequency and percentage. The Chi-square test 
or Fisher's exact test was used for the comparison of categorical variables between two groups. The independent $t$-test was used to compare normally distributed continuous data, and the Mann-Whitney U test was used to compare non-normally distributed continuous data. Multivariate analysis via multiple logistic regression model (backward stepwise) included all variables with a $p$-value less than 0.2 from univariate analysis. All statistical analyses were performed using SPSS Statistics (v18.0) software (SPSS, Inc., Chicago, IL, USA), and a 2 -sided $p$-value less than 0.05 was considered to be statistically significant.

\section{Results}

\subsection{Patient Data}

A total of 156 patients were enrolled. The mean age was 64 years, and $56.4 \%$ of patients were male. The most prevalent underlying conditions were diabetes mellitus $(32.1 \%)$, chronic cardiac disease $(28.2 \%)$, chronic kidney disease $(26.9 \%)$, and hematologic malignancies $(21.2 \%)$ (Table 1$)$.

Table 1. Characteristics of patients with candidemia caused by non-albicans Candida and Candida albicans.

\begin{tabular}{|c|c|c|c|c|}
\hline Characteristics & $\begin{array}{l}\text { Non-albicans Candida } \\
\quad(n=111)\end{array}$ & $\begin{array}{l}\text { C. albicans } \\
(n=45)\end{array}$ & $\begin{array}{l}\text { Odds Ratio } \\
\text { (95\% CI) }\end{array}$ & $p$ \\
\hline Male gender, $n(\%)$ & $58(52.2 \%)$ & $30(66.7 \%)$ & $0.54(0.27-1.13)$ & 0.1 \\
\hline Age (years), mean \pm SD & 63. \pm 18 & $67 \pm 18$ & - & 0.302 \\
\hline LOS (days), median (IQR) & $8(2-26)$ & $10(3-19)$ & - & 0.977 \\
\hline \multicolumn{5}{|l|}{ Diagnosis, $n(\%)$} \\
\hline Primary fungemia & $60(54.1 \%)$ & $19(42.2 \%)$ & $1.61(0.80-3.24)$ & 0.181 \\
\hline CRBSI & $41(36.9 \%)$ & $19(42.2 \%)$ & $0.80(0.40-1.62)$ & 0.539 \\
\hline Intra-abdominal infection & $7(6.3 \%)$ & $5(11.1 \%)$ & $0.54(0.16-1.80)$ & 0.329 \\
\hline Hepatosplenic abscess & $1(0.9 \%)$ & $0(0.0 \%)$ & - & 1 \\
\hline Endocarditis & $1(0.9 \%)$ & $0(0.0 \%)$ & - & 1 \\
\hline Urinary tract infection & $1(0.9 \%)$ & $2(4.4 \%)$ & $0.20(0.01-2.21)$ & 0.2 \\
\hline \multicolumn{5}{|l|}{ Comorbidities, $n(\%)$} \\
\hline Chronic cardiac disease & $30(27.0 \%)$ & $14(31.1 \%)$ & $0.82(0.38-1.75)$ & 0.608 \\
\hline Chronic lung disease & $15(13.5 \%)$ & $10(22.2 \%)$ & $0.55(0.22-1.33)$ & 0.179 \\
\hline Chronic kidney disease & $27(24.3 \%)$ & $15(33.3 \%)$ & $0.64(0.30-1.36)$ & 0.25 \\
\hline Chronic liver disease & $15(13.5 \%)$ & $1(2.2 \%)$ & $6.89(0.88-52.63)$ & 0.035 \\
\hline Diabetes mellitus & $37(33.3 \%)$ & $13(28.9 \%)$ & $1.23(0.58-2.62)$ & 0.59 \\
\hline HIV disease & $2(1.8 \%)$ & $1(2.2 \%)$ & $0.80(0.07-9.17)$ & 1 \\
\hline Autoimmune disease & $10(9.0 \%)$ & $5(11.1 \%)$ & $0.79(0.25-2.46)$ & 0.687 \\
\hline Hematologic malignancies & $28(25.2 \%)$ & $5(11.1 \%)$ & $2.69(0.97-7.51)$ & 0.051 \\
\hline HSCT & $2(1.8 \%)$ & $0(0.0 \%)$ & - & 1 \\
\hline Solid organ transplantation & $0(0.0 \%)$ & $1(2.2 \%)$ & - & 0.288 \\
\hline Mortality & $81(73.0 \%)$ & $30(66.7 \%)$ & $1.35(0.64-2.85)$ & 0.431 \\
\hline
\end{tabular}

A $p$-value < 0.05 indicates statistical significance; Abbreviations: SD, standard deviation; LOS, length of stay; IQR, interquartile range; CRBSI, catheter-related blood stream infection; HIV, human immunodeficiency virus; HSCT, hematopoietic stem cell transplantation.

\subsection{Candida Species Distribution and Antifungal Susceptibility}

The Candida species isolated from patient blood were C. tropicalis (49.4\%), C. albicans (28.8\%), C. glabrata (16.7\%), and C. parapsilosis (5.1\%) (Figure 1). Antifungal susceptibility results were available in 76 Candida isolates. Table 2 demonstrated the minimal inhibitory concentration (MIC) range, MIC50, and MIC90 of antifungal agents for each Candida species 
in this study. Fluconazole resistance was higher in C. tropicalis (37.8\%) than in C. albicans (14.3\%) (Figure 2).

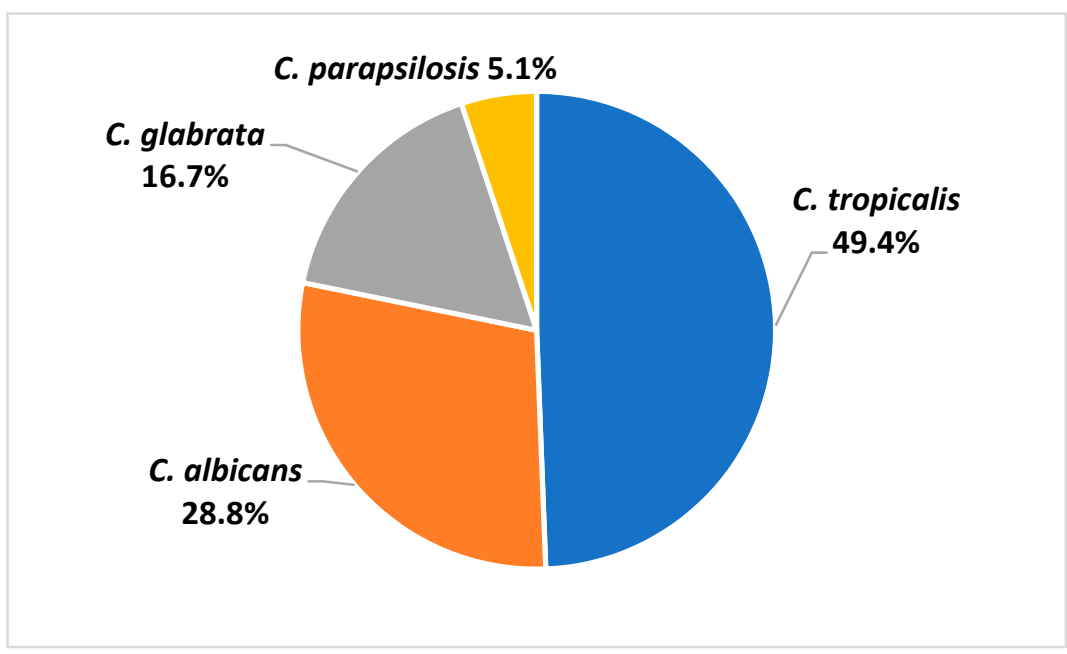

Figure 1. Species distribution of Candida isolated from blood samples of patients with candidemia.

Table 2. Minimal inhibitory concentration (MIC) range, MIC50, and MIC90 of antifungal agents for each Candida species.

\begin{tabular}{|c|c|c|c|c|}
\hline Species & Antifungal Agent & MIC Range (mg/L) & MIC50 (mg/L) & MIC90 (mg/L) \\
\hline \multirow{8}{*}{ Candida albicans } & Amphotericin B & $0.25-1$ & 0.5 & 1 \\
\hline & Fluconazole & $0.25-256$ & 0.25 & 1 \\
\hline & Itraconazole & $0.025-0.25$ & 0.06 & 0.12 \\
\hline & Voriconazole & $\leq 0.008-2$ & $\leq 0.008$ & 0.03 \\
\hline & Posaconazole & $\leq 0.008-0.25$ & 0.03 & 0.06 \\
\hline & 5-fluorocytosine & $\leq 0.06-0.12$ & $\leq 0.06$ & 0.12 \\
\hline & Caspofungin & $0.03-0.06$ & 0.06 & 0.06 \\
\hline & Micafungin & $\leq 0.008-0.03$ & $\leq 0.008$ & 0.02 \\
\hline \multirow{8}{*}{ Candida tropicalis } & Amphotericin B & $0.5-2$ & 1 & 1 \\
\hline & Fluconazole & $\leq 0.12-256$ & 2 & 256 \\
\hline & Itraconazole & $0.06-1$ & 0.25 & 1 \\
\hline & Voriconazole & $0.06-4$ & 0.12 & 0.5 \\
\hline & Posaconazole & $0.06-1$ & 0.25 & 1 \\
\hline & 5-fluorocytosine & $\leq 0.06-0.25$ & $\leq 0.06$ & 0.06 \\
\hline & Caspofungin & $0.03-0.25$ & 0.03 & 0.12 \\
\hline & Micafungin & $0.03-0.5$ & 0.03 & 0.03 \\
\hline \multirow{8}{*}{ Candida parapsilosis } & Amphotericin B & $0.5-1$ & 1 & 1 \\
\hline & Fluconazole & $0.25-8$ & 1 & 8 \\
\hline & Itraconazole & $0.06-0.12$ & 0.06 & 0.12 \\
\hline & Voriconazole & $\leq 0.008-0.25$ & 0.02 & 0.25 \\
\hline & Posaconazole & $0.03-0.06$ & 0.03 & 0.06 \\
\hline & 5-fluorocytosine & $\leq 0.06-0.12$ & 0.06 & 0.12 \\
\hline & Caspofungin & $0.25-0.5$ & 0.25 & 0.5 \\
\hline & Micafungin & $0.5-1$ & 0.5 & 1 \\
\hline
\end{tabular}


Table 2. Cont.

\begin{tabular}{|c|c|c|c|c|}
\hline Species & Antifungal Agent & MIC Range (mg/L) & MIC50 (mg/L) & MIC90 (mg/L) \\
\hline \multirow{8}{*}{ Candida glabrata } & Amphotericin B & $0.5-2$ & 1 & 1 \\
\hline & Fluconazole & $2-32$ & 8 & 32 \\
\hline & Itraconazole & $0.25-1$ & 0.5 & 1 \\
\hline & Voriconazole & $0.12-1$ & 0.25 & 1 \\
\hline & Posaconazole & $0.25-2$ & 1 & 2 \\
\hline & 5-fluorocytosine & $\leq 0.06$ & $\leq 0.06$ & $\leq 0.06$ \\
\hline & Caspofungin & $0.03-0.25$ & 0.06 & 0.12 \\
\hline & Micafungin & $\leq 0.008-0.03$ & 0.03 & 0.03 \\
\hline
\end{tabular}

120

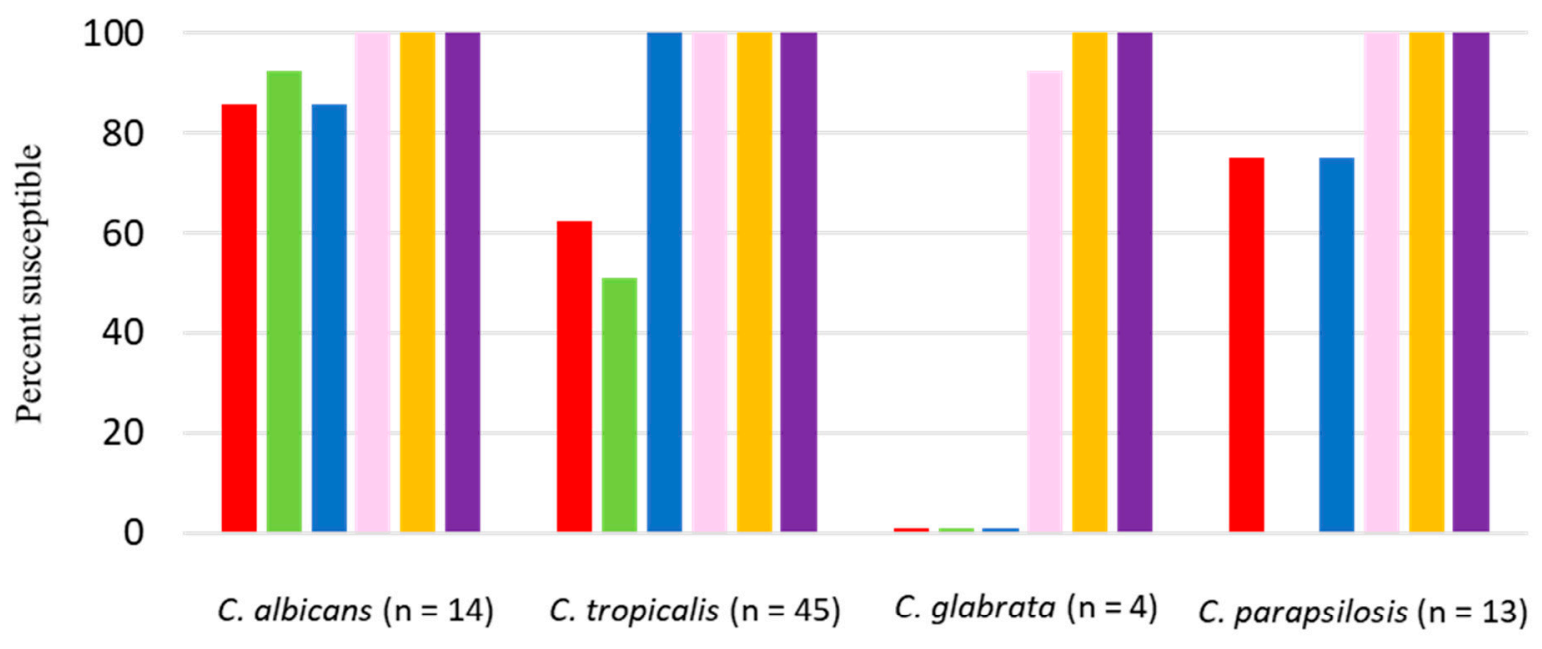

Candida species

Fluconazole $\square$ Itraconazole $\square$ Voriconazole
Caspofungin $\square$ Micafungin $\square$ Amphotericin B

Figure 2. Antifungal susceptibility of Candida isolates.

3.3. Factors Significantly and Independently Associated with Non-albicans Candida Candidemia

Patient characteristics and other factors significantly associated with non-albicans Candida and C. albicans candidemia are shown in Tables 1 and 3. Chronic liver disease and neutropenia were the only two factors found to be significantly associated with non-albicans Candida candidemia in univariate analysis.

Subgroup analysis of candidemia caused by C. tropicalis or C. albicans to identify the factors associated with those two infections is shown in Tables 4 and 5. Multivariate analysis revealed male gender, chronic liver disease, and neutropenia to be factors independently associated with C. tropicalis infection (Table 6). The mortality rate was not significantly different among patients with candidemia caused by C. albicans, non-albicans Candida, or C. tropicalis. 
Table 3. Univariate analysis for factors significantly associated with non-albicans Candida and Candida albicans infection in patients with candidemia.

\begin{tabular}{|c|c|c|c|c|}
\hline Risk Factors & $\begin{array}{l}\text { Non-albicans Candida } \\
\quad(n=111), n(\%)\end{array}$ & $\begin{array}{c}\text { C. albicans } \\
(n=45), n(\%)\end{array}$ & $\begin{array}{l}\text { Odds Ratio } \\
\text { (95\% CI) }\end{array}$ & $p$ \\
\hline Central venous catheterization & $73(65.8 \%)$ & $33(73.3 \%)$ & $0.70(0.32-1.51)$ & 0.359 \\
\hline Mechanical ventilator & $81(73.0 \%)$ & $36(80.0 \%)$ & $0.68(0.29-1.56)$ & 0.358 \\
\hline Parenteral nutrition & $36(32.4 \%)$ & $12(26.7 \%)$ & $1.32(0.61-2.86)$ & 0.480 \\
\hline Hemodialysis & $46(41.4 \%)$ & $18(40.0 \%)$ & $1.06(0.52-2.15)$ & 0.868 \\
\hline Peritoneal dialysis & $3(2.7 \%)$ & $0(0.0 \%)$ & - & 0.557 \\
\hline Recent abdominal surgery & $19(17.1 \%)$ & $7(15.6 \%)$ & $1.12(0.44-2.88)$ & 0.813 \\
\hline ICU admission & $65(58.6 \%)$ & $25(55.6 \%)$ & $1.13(0.56-2.27)$ & 0.731 \\
\hline Urinary catheterization & $93(83.6 \%)$ & $40(88.9 \%)$ & $0.65(0.22-1.86)$ & 0.415 \\
\hline Presence of prosthesis & $12(10.8 \%)$ & $9(20.0 \%)$ & $0.48(0.19-1.25)$ & 0.128 \\
\hline Carbapenem use & $59(53.2 \%)$ & $21(46.7 \%)$ & $1.30(0.65-2.30)$ & 0.463 \\
\hline Cephalosporin use & $18(16.2 \%)$ & $8(17.8 \%)$ & $0.90(0.36-2.24)$ & 0.813 \\
\hline Fluoroquinolone use & $13(11.7 \%)$ & $9(20.0 \%)$ & $0.53(0.21-1.35)$ & 0.178 \\
\hline Corticosteroid use & $12(10.8 \%)$ & $7(15.6 \%)$ & $0.66(0.24-1.80)$ & 0.412 \\
\hline Neutropenia & $25(22.5 \%)$ & $4(8.9 \%)$ & $2.97(0.97-9.09)$ & 0.047 \\
\hline Presence of yeast in urine & $56(50.5 \%)$ & $23(51.1 \%)$ & $0.91(0.42-1.96)$ & 0.816 \\
\hline Presence of yeast in sputum & $49(44.1 \%)$ & $24(53.3 \%)$ & $0.68(0.31-1.51)$ & 0.342 \\
\hline Presence of yeast in feces & $6(5.4 \%)$ & $1(2.2 \%)$ & $2.07(0.22-19.23)$ & 0.517 \\
\hline Antifungal exposure within 1 month & $4(3.6 \%)$ & $2(4.4 \%)$ & $0.80(0.14-4.55)$ & 1.000 \\
\hline Azoles exposure within 1 month & $4(3.6 \%)$ & $1(2.2 \%)$ & $1.64(0.18-15.15)$ & 1.000 \\
\hline
\end{tabular}

A $p$-value $<0.05$ indicates statistical significance; Abbreviation: CI, confidence interval; ICU, intensive care unit.

Table 4. Characteristics of patients with candidemia caused by Candida tropicalis or Candida albicans.

\begin{tabular}{|c|c|c|c|c|}
\hline Factors & $\begin{array}{l}\text { C. tropicalis } \\
\quad(n=77)\end{array}$ & $\begin{array}{c}\text { C. albicans } \\
(n=45)\end{array}$ & $\begin{array}{l}\text { Odds Ratio } \\
(95 \% \text { CI })\end{array}$ & $p$ \\
\hline Male gender, $n(\%)$ & $58(75.3 \%)$ & $30(66.7 \%)$ & $1.95(0.91-4.18)$ & 0.085 \\
\hline Age (years), mean $\pm S D$ & $62 \pm 18$ & $67 \pm 18$ & - & 0.302 \\
\hline LOS (days), median (IQR) & $7(2-18)$ & $10(3-19)$ & - & 0.977 \\
\hline \multicolumn{5}{|l|}{ Diagnosis, $n(\%)$} \\
\hline Primary fungemia & $42(54.5 \%)$ & $19(42.2 \%)$ & $1.64(0.78-3.45)$ & 0.181 \\
\hline CRBSI & $30(39.0 \%)$ & $19(42.2 \%)$ & $0.87(0.41-1.85)$ & 0.539 \\
\hline Intra-abdominal infection & $2(2.6 \%)$ & $5(11.1 \%)$ & $0.21(0.04-1.15)$ & 0.329 \\
\hline Hepatosplenic abscess & $1(1.3 \%)$ & $0(0.0 \%)$ & - & 1 \\
\hline Endocarditis & $1(1.3 \%)$ & $0(0.0 \%)$ & - & 1 \\
\hline Urinary tract infection & $1(1.3 \%)$ & $2(4.4 \%)$ & $0.28(0.03-3.21)$ & 0.2 \\
\hline \multicolumn{5}{|l|}{ Comorbidities, $n(\%)$} \\
\hline Chronic cardiac disease & $20(26.0 \%)$ & $14(31.1 \%)$ & $0.78(0.35-1.75)$ & 0.541 \\
\hline Chronic lung disease & $10(13.0 \%)$ & $10(22.2 \%)$ & $0.52(0.20-1.37)$ & 0.184 \\
\hline Chronic kidney disease & $17(22.1 \%)$ & $15(33.3 \%)$ & $0.57(0.25-1.29)$ & 0.173 \\
\hline Chronic liver disease & $13(16.9 \%)$ & $1(2.2 \%)$ & $8.94(1.13-70.82)$ & 0.014 \\
\hline Diabetes mellitus & $23(29.9 \%)$ & $13(28.9 \%)$ & $1.05(0.48-2.35)$ & 0.909 \\
\hline HIV disease & $2(2.6 \%)$ & $1(2.2 \%)$ & $1.17(0.10-13.32)$ & 1 \\
\hline
\end{tabular}


Table 4. Cont.

\begin{tabular}{|c|c|c|c|c|}
\hline Factors & $\begin{array}{l}\text { C. tropicalis } \\
\quad(n=77)\end{array}$ & $\begin{array}{l}\text { C. albicans } \\
(n=45)\end{array}$ & $\begin{array}{l}\text { Odds Ratio } \\
\text { (95\% CI) }\end{array}$ & $p$ \\
\hline Autoimmune disease & $8(10.4 \%)$ & $5(11.1 \%)$ & $0.93(0.28-3.03)$ & 1 \\
\hline Hematologic malignancies & $26(33.8 \%)$ & $5(11.1 \%)$ & $4.08(1.44-11.57)$ & 0.006 \\
\hline HSCT & $2(2.6 \%)$ & $0(0.0 \%)$ & - & 1 \\
\hline Solid organ transplantation & $0(0.0 \%)$ & $1(2.2 \%)$ & - & 0.288 \\
\hline Mortality & $59(76.7 \%)$ & $30(66.7 \%)$ & $1.64(0.73-3.70)$ & 0.232 \\
\hline
\end{tabular}

\footnotetext{
A $p$-value < 0.05 indicates statistical significance; Abbreviations: CI, confidence interval; SD, standard deviation; LOS, length of stay; IQR, interquartile range; CRBSI, catheter-related blood stream infection; HIV, human immunodeficiency virus; HSCT, hematopoietic stem cell transplantation.
}

Table 5. Univariate analysis for factors significantly associated with Candida tropicalis and Candida albicans infection in patients with candidemia.

\begin{tabular}{|c|c|c|c|c|}
\hline Risk Factors & $\begin{array}{c}\text { C. tropicalis } \\
(n=77), n(\%)\end{array}$ & $\begin{array}{c}\text { C. albicans } \\
(n=45), n(\%)\end{array}$ & $\begin{array}{l}\text { Odds Ratio } \\
\text { (95\% CI) }\end{array}$ & $p$ \\
\hline Central venous catheterization & $49(63.6 \%)$ & $33(73.3 \%)$ & $0.64(0.28-1.43)$ & 0.271 \\
\hline Mechanical ventilator & $53(68.8 \%)$ & $36(80.0 \%)$ & $0.55(0.23-1.33)$ & 0.180 \\
\hline Parenteral nutrition & $22(28.6 \%)$ & $12(26.7 \%)$ & $1.10(0.48-2.51)$ & 0.821 \\
\hline Hemodialysis & $34(44.2 \%)$ & $18(40.0 \%)$ & $1.19(0.56-2.50)$ & 0.654 \\
\hline Peritoneal dialysis & - & - & - & - \\
\hline Recent abdominal surgery & $11(14.3 \%)$ & $7(15.6 \%)$ & $0.91(0.32-2.53)$ & 0.849 \\
\hline ICU admission & $45(58.4 \%)$ & $25(55.6 \%)$ & $1.13(0.54-2.36)$ & 0.756 \\
\hline Urinary catheterization & $63(81.8 \%)$ & $40(88.9 \%)$ & $0.56(0.19-1.68)$ & 0.299 \\
\hline Presence of prosthesis & $7(9.1 \%)$ & $9(20.0 \%)$ & $0.40(0.14-1.16)$ & 0.085 \\
\hline Carbapenem use & $41(53.2 \%)$ & $21(46.7 \%)$ & $1.30(0.62-2.72)$ & 0.483 \\
\hline Cephalosporin use & $9(11.7 \%)$ & $8(17.8 \%)$ & $0.61(0.22-1.72)$ & 0.349 \\
\hline Fluoroquinolone use & $8(10.4 \%)$ & $9(20.0 \%)$ & $0.46(0.17-1.30)$ & 0.139 \\
\hline Corticosteroid use & $9(11.7 \%)$ & $7(15.6 \%)$ & $0.72(0.25-2.08)$ & 0.541 \\
\hline Neutropenia & $23(29.9 \%)$ & $4(8.9 \%)$ & $4.37(1.40-13.61)$ & 0.007 \\
\hline Presence of yeast in urine & $40(51.9 \%)$ & $23(51.1 \%)$ & $0.93(0.41-2.10)$ & 0.864 \\
\hline Presence of yeast in sputum & $35(45.4 \%)$ & $24(53.3 \%)$ & $0.79(0.34-1.85)$ & 0.587 \\
\hline Presence of yeast in feces & $6(7.8 \%)$ & $1(2.2 \%)$ & $3.16(0.33-30.00)$ & 0.400 \\
\hline Antifungal exposure within 1 month & $4(5.1 \%)$ & $2(4.4 \%)$ & $1.18(0.21-6.70)$ & 1.000 \\
\hline Azoles exposure within 1 month & $3(3.9 \%)$ & $1(2.2 \%)$ & $1.78(0.18-17.68)$ & 1.000 \\
\hline
\end{tabular}

A $p$-value $<0.05$ indicates statistical significance; Abbreviation: CI, confidence interval; ICU, intensive care unit.

Table 6. Multivariate analysis for factors independently associated with Candida tropicalis and Candida albicans infection in patients with candidemia.

\begin{tabular}{|c|c|c|c|c|}
\hline Risk Factors & $\begin{array}{l}\text { C. tropicalis } \\
(n=77), n(\%)\end{array}$ & $\begin{array}{c}\text { C. albicans } \\
(n=45), n(\%)\end{array}$ & $\begin{array}{c}\text { Adjusted OR } \\
(95 \% \text { CI })\end{array}$ & $p$ \\
\hline Male gender & $58(75.3 \%)$ & $30(66.7 \%)$ & $2.34(1.04-5.29)$ & 0.04 \\
\hline Chronic liver disease & $13(16.9 \%)$ & $1(2.2 \%)$ & $11.39(1.38-94.02)$ & 0.024 \\
\hline Neutropenia & $23(29.9 \%)$ & $4(8.9 \%)$ & $4.31(1.34-13.87)$ & 0.014 \\
\hline Intra-abdominal infection & $2(2.6 \%)$ & $5(11.1 \%)$ & $0.36(0.07-2.05)$ & 0.252 \\
\hline Received fluoroquinolones at least 7 days & $8(10.4 \%)$ & $9(20.0 \%)$ & $0.53(0.17-1.63)$ & 0.266 \\
\hline
\end{tabular}


Table 6. Cont.

\begin{tabular}{|c|c|c|c|c|}
\hline Risk Factors & $\begin{array}{c}\text { C. tropicalis } \\
(n=77), n(\%)\end{array}$ & $\begin{array}{c}\text { C. albicans } \\
(n=45), n(\%)\end{array}$ & $\begin{array}{c}\text { Adjusted OR } \\
(95 \% \text { CI })\end{array}$ & $p$ \\
\hline Presence of prosthesis & $7(9.1 \%)$ & $9(20.0 \%)$ & $0.61(0.19-1.94)$ & 0.404 \\
\hline Chronic kidney disease & $17(22.1 \%)$ & $15(33.3 \%)$ & $0.76(0.304-1.92)$ & 0.566 \\
\hline Hematologic malignancies & $26(33.8 \%)$ & $5(11.1 \%)$ & $1.27(0.25-6.47)$ & 0.774 \\
\hline Chronic lung disease & $10(13.0 \%)$ & $10(22.2 \%)$ & $0.92(0.27-3.10)$ & 0.893 \\
\hline Mechanical ventilator & $53(68.8 \%)$ & $36(80.0 \%)$ & $1.03(0.37-2.85)$ & 0.958 \\
\hline Primary fungemia & $42(54.5 \%)$ & $19(42.2 \%)$ & $1.02(0.42-2.50)$ & 0.958 \\
\hline
\end{tabular}

A $p$-value $<0.05$ indicates statistical significance; Abbreviation: OR, odds ratio; $\mathrm{CI}$, confidence interval.

\section{Discussion}

From January 2016 to December 2017, we analyzed data from 156 candidemia patients admitted to Siriraj Hospital, the largest university hospital in Thailand. Most patients had candidemia that was caused by non-albicans Candida (71.2\%), which is consistent with a previous study in Thailand, and which reveals an increasing incidence of non-albicans candidemia [4]. C. tropicalis was found to be the most common Candida species in patients with candidemia, and this is similar to the results of previous studies conducted at the same hospital $[5,19]$. It was proposed that the pathogenicity of $C$. tropicalis may be associated with a warmer climate [12], which may explain why it was found to be the most common Candida species in our study.

The present study identified neutropenia and chronic liver disease as potential risk factors for non-albicans Candida candidemia. A previous study also reported non-albicans Candida candidemia to be associated with neutropenic patients [6]. However, no previous study has reported chronic liver disease as a risk factor of non-albicans Candida candidemia.

Risk factors associated with $C$. tropicalis and $C$. albicans candidemia were also analyzed in this study. The results of that analysis revealed neutropenia, chronic liver disease, and male gender to be independent risk factors for $C$. tropicalis candidemia. A previous study reported the independent risk factors for $C$. tropicalis candidemia to be abdomen portal entry, hematologic malignancies, and older age [20]. Our study confirmed that C. tropicalis seems to originate from the intra-abdominal origin, and it tends to infect neutropenic patients.

Candida tropicalis has become increasingly resistant to fluconazole. Two previous studies that were conducted at our center in 2009 and 2013 found rates of C. tropicalis susceptibility to fluconazole of $100 \%$ and $70.3 \%$, respectively $[5,19]$. However, in this study, we found a rate of $C$. tropicalis susceptibility to fluconazole of only $62 \%$. Therefore, empirical antifungal treatment with echinocandins or amphotericin B product in candidemia patients with risk factors for $C$. tropicalis infection is strongly recommended. In our study, most of the patients infected with fluconazole-resistant $C$. tropicalis did not have a history of azole exposure and they may be acquired from the environment [21]. Azoles have been used in agriculture in Thailand and the link between fluconazole-resistant Candida and environmental azole exposure needs to be investigated.

The mortality rate among candidemia patients in this study was approximately $70 \%$, which is higher than in other studies, but the mortality rate was not found to be significantly different between C. albicans and non-albicans Candida candidemia. This extremely high mortality rate in candidemia patients highlights the need to improve candidemia management guidelines in Thailand.

The limitations of this study include its single-center retrospective cohort design, the fact that the epidemiology of Candida species can differ among hospitals, and that antifungal susceptibility testing was not routinely performed in every included study patient. 


\section{Conclusions}

In candidemia patients, chronic liver disease, neutropenia, and male gender were found to be independently associated with $C$. tropicalis infection. Due to the observed high resistance of $C$. tropicalis to fluconazole, fluconazole should not be used for empirical antifungal treatment in these patients.

Author Contributions: Conceptualization, C.N. and M.C.; methodology, C.N.,P.C. and M.C.; software, C.N. and A.W.; validation, C.N., P.C. and M.C.; formal analysis, C.N. and M.C.; investigation, M.C.; resources, P.C. and A.W.; data curation, C.N.; writing-original draft preparation, C.N.; writing-review and editing, M.C.; visualization, C.N. and M.C.; supervision, M.C.; project administration, A.W.; funding acquisition, P.C. and M.C. All authors have read and agreed to the published version of the manuscript.

Funding: Methee Chayakulkeeree and Piriyaporn Chongtrakool received Chalermphrakiat Grants from the Faculty of Medicine Siriraj Hospital. Mahidol University.

Institutional Review Board Statement: The study was conducted according to the guidelines of the Declaration of Helsinki and approved by the Siriraj Institutional Review Board (SIRB) (COA no. Si $517 / 2018)$.

Informed Consent Statement: Patient consent was waived due to the confidential retrospective nature of this study.

Data Availability Statement: No new data were created or analyzed in this study. Data sharing is not applicable to this article.

Acknowledgments: The authors gratefully acknowledge Khemajira Karaketklang, MPH, Division of Research and Academics, Department of Medicine, Faculty of Medicine Siriraj Hospital, Mahidol University for her assistance with statistical analysis; and staff members from the laboratory of the Department of Microbiology for their assistance collecting candidemia patient data.

Conflicts of Interest: The authors declare no conflict of interest.

\section{References}

1. Perlroth, J.; Choi, B.; Spellberg, B. Nosocomial fungal infections: Epidemiology, diagnosis, and treatment. Med. Mycol. 2007, 45, 321-346. [CrossRef] [PubMed]

2. Sardi, J.C.O.; Scorzoni, L.; Bernardi, T.; Fusco-Almeida, A.M.; Mendes Giannini, M.J.S. Candida species: Current epidemiology, pathogenicity, biofilm formation, natural antifungal products and new therapeutic options. J. Med. Microbiol. 2013, 62, 10-24. [CrossRef] [PubMed]

3. Chayakulkeeree, M.; Denning, D.W. Serious fungal infections in Thailand. Eur. J. Clin. Microbiol. Infect. Dis. 2017, 36, 931-935. [CrossRef] [PubMed]

4. Chaiwarith, R.; Ounbang, P.; Khamwan, C.; Nuntachit, N.; Sirisanthana, T.; Supparatpinyo, K. Epidemiology of adult candidemia at Chiang Mai University Hospital. Southeast Asian J. Trop. Med. Public Health 2011, 42, 1505-1514. [PubMed]

5. Boonyasiri, A.; Jearanaisilavong, J.; Assanasen, S. Candidemia in Siriraj Hospital: Epidemiology and factors associated with mortality. J. Med. Assoc. Thail. 2013, 96 (Suppl. 2), S91-S97.

6. Chi, H.W.; Yang, Y.S.; Shang, S.T.; Chen, K.H.; Yeh, K.M.; Chang, F.Y.; Lin, J.C. Candida albicans versus non-albicans bloodstream infections: The comparison of risk factors and outcome. J. Microbiol. Immunol. Infect. 2011, 44, 369-375. [CrossRef]

7. Oberoi, J.K.; Wattal, C.; Goel, N.; Raveendran, R.; Datta, S.; Prasad, K. Non-albicans Candida species in blood stream infections in a tertiary care hospital at New Delhi, India. Indian J. Med. Res. 2012, 136, 997-1003.

8. Kaur, R.D.M.; Goyal, R.; Kumar, R. Emergence of non-albicans Candida species and antifungal resistance in intensivecare unit patients. Asian Pac. J. Trop. Biomed. 2016, 6, 455-460. [CrossRef]

9. Zakhem, A.E.; Istambouli, R.; Alkozah, M.; Gharamti, A.; Tfaily, M.A.; Jabbour, J.F.; Araj, G.F.; Tamim, H.; Kanj, S.S. Predominance of Candida glabrata among non-albicans Candida species in a 16-year study of candidemia at a tertiary care center in Lebanon. Pathogens 2021, 10, 82. [CrossRef]

10. Zhang, W.; Song, X.; Wu, H.; Zheng, R. Epidemiology, species distribution, and predictive factors for mortality of candidemia in adult surgical patients. BMC Infect. Dis. 2020, 20, 506. [CrossRef]

11. Lortholary, O.; Renaudat, C.; Sitbon, K.; Desnos-Ollivier, M.; Bretagne, S.; Dromer, F.; French Mycoses Study Group. The risk and clinical outcome of candidemia depending on underlying malignancy. Intensive Care Med. 2017, 43, 652-662. [CrossRef] [PubMed]

12. Tan, B.H.; Chakrabarti, A.; Li, R.Y.; Patel, A.K.; Watcharananan, S.P.; Liu, Z.; Chindamporn, A.; Tan, A.L.; Sun, P.L.; Wu, U.I.; et al. Incidence and species distribution of candidaemia in Asia: A laboratory-based surveillance study. Clin. Microbiol. Infect. 2015, 21, 946-953. [CrossRef] [PubMed] 
13. Collin, B.; Clancy, C.J.; Nguyen, M.H. Antifungal resistance in non- albicans Candida species. Drug Resist. Updat. 1999, 2, 9-14. [CrossRef]

14. Fernandez-Ruiz, M.; Puig-Asensio, M.; Guinea, J.; Almirante, B.; Padilla, B.; Almela, M.; Diaz-Martin, A.; Rodriguez-Bano, J.; Cuenca-Estrella, M.; Aguado, J.M.; et al. Candida tropicalis bloodstream infection: Incidence, risk factors and outcome in a population-based surveillance. J. Infect. 2015, 71, 385-394. [CrossRef] [PubMed]

15. Lortholary, O.; Renaudat, C.; Sitbon, K.; Madec, Y.; Denoeud-Ndam, L.; Wolff, M.; Fontanet, A.; Bretagne, S.; Dromer, F.; French Mycosis Study Group. Worrisome trends in incidence and mortality of candidemia in intensive care units (Paris area, 2002-2010). Intensive Care Med. 2014, 40, 1303-1312. [CrossRef] [PubMed]

16. Kothalawala, M.; Jayaweera, J.; Arunan, S.; Jayathilake, A. The emergence of non-albicans candidemia and evaluation of HiChrome Candida differential agar and VITEK2 YST(R) platform for differentiation of Candida bloodstream isolates in teaching hospital Kandy, Sri Lanka. BMC Microbiol. 2019, 19, 136. [CrossRef]

17. Ostrosky-Zeichner, L.; Kullberg, B.J.; Bow, E.J.; Hadley, S.; Leon, C.; Nucci, M.; Patterson, T.F.; Perfect, J.R. Early treatment of candidemia in adults: A review. Med. Mycol. 2011, 49, 113-120. [CrossRef]

18. Garey, K.W.; Rege, M.; Pai, M.P.; Mingo, D.E.; Suda, K.J.; Turpin, R.S.; Bearden, D.T. Time to initiation of fluconazole therapy impacts mortality in patients with candidemia: A multi-institutional study. Clin. Infect. Dis. 2006, 43, 25-31. [CrossRef]

19. Tan, T.Y.; Hsu, L.Y.; Alejandria, M.M.; Chaiwarith, R.; Chinniah, T.; Chayakulkeeree, M.; Choudhury, S.; Chen, Y.H.; Shin, J.H.; Kiratisin, P.; et al. Antifungal susceptibility of invasive Candida bloodstream isolates from the Asia-Pacific region. Med. Mycol. 2016, 54, 471-477. [CrossRef]

20. Munoz, P.; Giannella, M.; Fanciulli, C.; Guinea, J.; Valerio, M.; Rojas, L.; Rodriguez-Creixems, M.; Bouza, E. Candida tropicalis fungaemia: Incidence, risk factors and mortality in a general hospital. Clin. Microbiol. Infect. 2011, 17, 1538-1545. [CrossRef]

21. Wang, Q.; Tang, D.; Tang, K.; Guo, J.; Huang, Y.; Li, C. Multilocus sequence typing reveals clonality of fluconazole-nonsusceptible Candida tropicalis: A study from Wuhan to the global. Front. Microbiol. 2020, 11, 554249. [CrossRef] [PubMed] 